\title{
MRI characterization of peripheral arterial chronic total occlusions at 7 Tesla with microCT and histologic validation
}

\author{
Trisha Roy ${ }^{*}$, Garry Liu, Xiuling Qi, Andrew Dueck, Graham A Wright \\ From 18th Annual SCMR Scientific Sessions \\ Nice, France. 4-7 February 2015
}

\section{Background}

Current treatment guidelines recommend surgical bypass for peripheral chronic total occlusions (CTOs)[1]. Percutaneous transluminal angioplasty (PTA), however, offers a less invasive approach with improved perioperative morbidity, shorter length of hospital stay, and lower cost[2]. Not all lesions are amenable to this technique and there is a significant primary failure rate[2]. Predicting lesion crossability is difficult because current imaging techniques offer limited information with which to characterize CTOs. The ability to distinguish between hard and soft biological substances that compose a CTO can aid procedural planning and facilitate intervention. This pilot study demonstrates the ability of high resolution MRI to characterize peripheral CTO components with microCT and histologic validation.

\section{Methods}

MRI was performed on 10 excised human peripheral arterial CTO segments from 3 patients. Each sample was imaged at $7 \mathrm{~T}$ (Bruker BioSpec preclinical MR imaging system) at high resolution $(75 \times 75 \times 75 \mu \mathrm{m}$ voxels) to produce three-dimensional $\mathrm{T} 2$ - and $\mathrm{T} 2$ *weighted images. For T2-weighted imaging, a spinecho sequence with an echo-train length of 8 and an equivalent TE of $37 \mathrm{~ms}$ was used. For T2*-imaging, an ultrashort echo (UTE) sequence was used with a set of echo times: $\{20 \mu \mathrm{s}, 500 \mu \mathrm{s}, 1 \mathrm{~ms}\}$. A difference image was produced by subtracting the complex signal values of the $1 \mathrm{~ms}$-image from those of the $20 \mu \mathrm{s}$-image. An offresonance map was produced using linear fits of the phase signals of the set of $\mathrm{T} 2 *$ images. The T2-weighted image, T2*-difference-image, and off-resonance map were used together to differentiate between lesion components. Each sample was imaged with microCT at high resolution ( $5 \times 5 \times 5 \mu$ m voxels) to identify calcium. Samples were then decalcified during histologic processing. H\&E staining was used to identify microlumina, smooth muscle cells, blood, and residual calcium. Movat's pentachrome staining was used to identify elastic lamina, fibrin, collagen, cholesterol clefts, and proteoglycan.

Table 1 CTO components with corresponding imaging signatures

\begin{tabular}{cccc}
\hline CTO component (in order of descending hardness [3]) & T2 weighted & T2*-difference & Resonance shift \\
\hline Calcium & Black & Black & $0-500 \mathrm{~Hz}$ \\
\hline Collagen & Black & Hyperintense & $1 \mathrm{kHz}$ \\
\hline Cholesterol clefts & Dark grey & Dark & $0-500 \mathrm{~Hz}$ \\
\hline Loose fibrotic tissue & Light grey & Dark & $0-500 \mathrm{~Hz}$ \\
\hline Lipid & Hyperintense & Variable & Variable \\
\hline Blood/thrombus & Black & Hyperintense & $0-500 \mathrm{~Hz}$ \\
\hline
\end{tabular}

Sunnybrook Research Institute, University of Toronto, Toronto, ON, Canada 


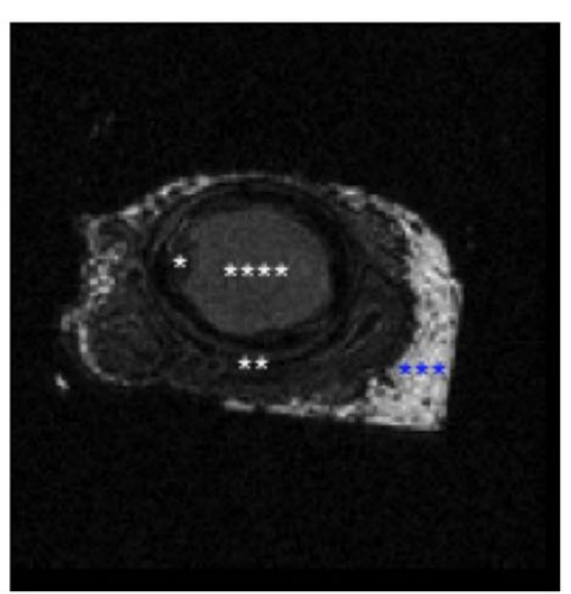

(a) T2 weighted image

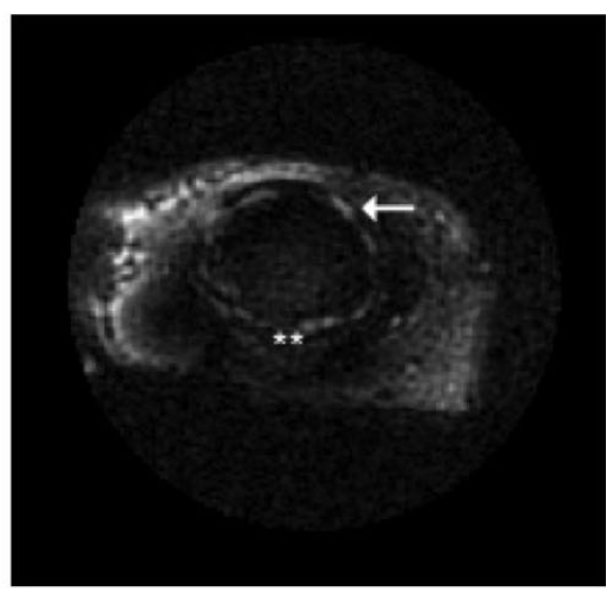

(b) $\mathrm{T} 2 *$-difference image
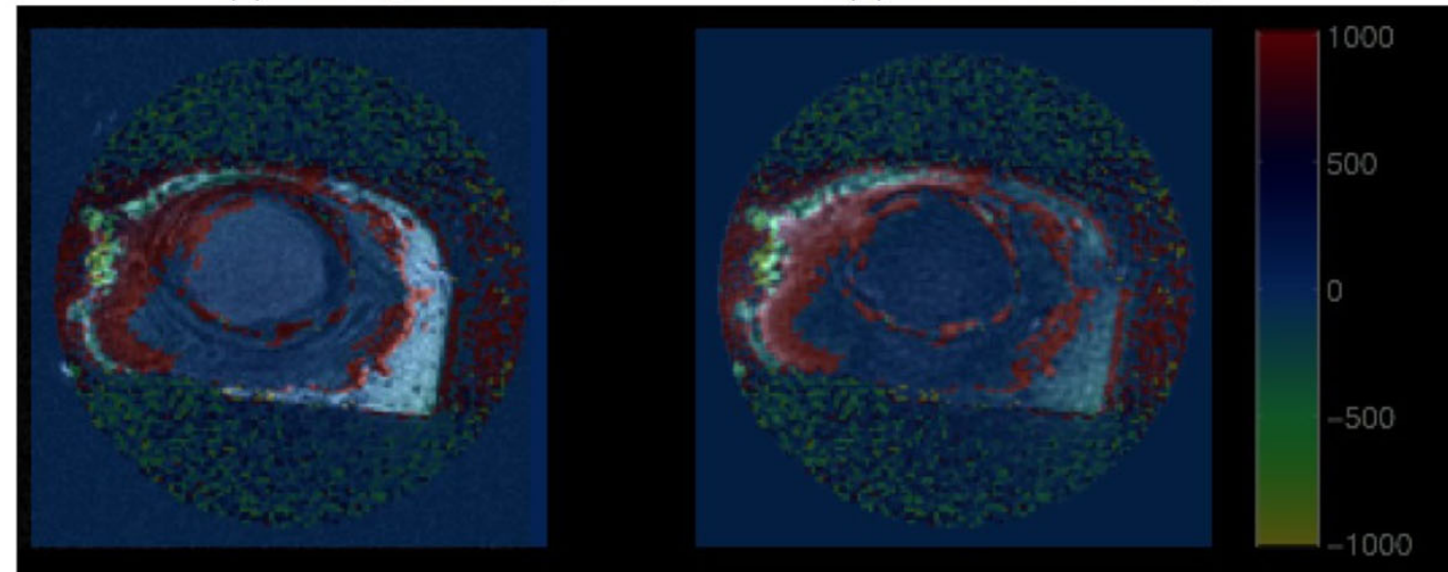

(c) T2 weighted and T2*-difference image with off-resonance map colour overlay. Red areas correspond to a $1 \mathrm{kHz}$ off-resonance frequency as indicated by the colour bar.

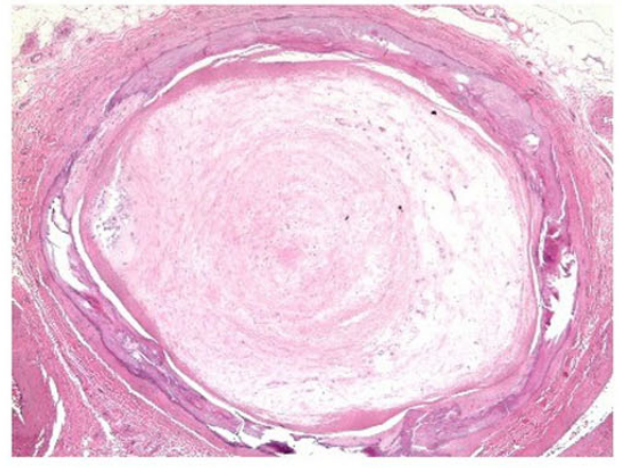

(d) Histology with H\&E stain

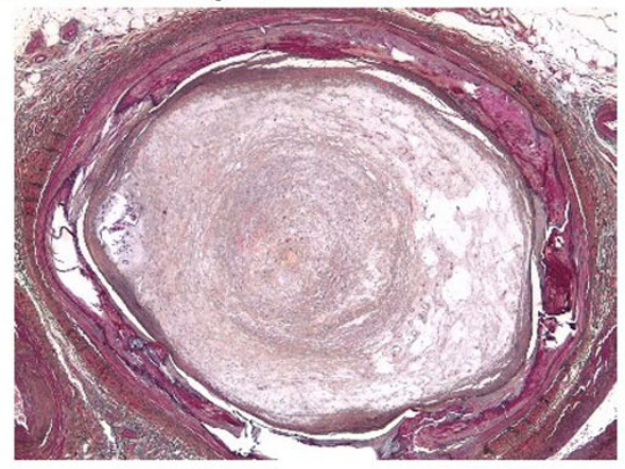

(e) Histology with Movat's pentachrome stain

Figure 1 Human peripheral arterial chronic total occlusion. The T2 weighted image shows soft lesion components in relative grey scale. Lipid/adipose tissue appears hyperintense $\left(^{* *}\right)$, loose fibrotic tissue appears grey $\left(^{* * *}\right)$ and cholesterol clefts appear dark grey $\left(^{*}\right)$. Hard lesion components like collagen are not seen on T2 weighted images but appears as hyperintense (arrow) on the T2*-difference image. Collagen is also highlighted on the off-resonance map at a $1 \mathrm{kHz}$ off-resonance frequency[4], designated in the colour overlay as red. This collagen imaging signature corresponds to the yellow ring on the Movat's pentachrome stain encircling the CTO core. Calcium is shown as a dark ring $\left(^{* *}\right)$ on both the $\mathrm{T} 2$ weighted and the T2*-difference images. 


\section{Results}

Hard and soft regions of interest (Table 1) were identified on the T2-weighted, T2*-difference, and off-resonance images with good correlation with microCT and histology (Figure 1).

\section{Conclusions}

These preliminary results demonstrate the potential of high-resolution T2 and T2* imaging using UTE to characterize hard and soft lesion components in human peripheral CTOs. This provides the foundation for further work in determining the lesion crossability and procedural success rates in CTOs.

\section{Funding}

Canadian Institutes of Health Research.

Published: 3 February 2015

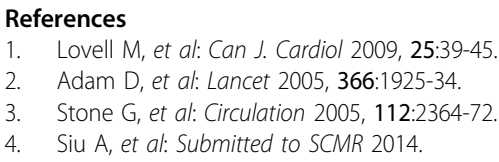

- Convenient online submission

- Thorough peer review

- No space constraints or color figure charges

- Immediate publication on acceptance

- Inclusion in PubMed, CAS, Scopus and Google Scholar

- Research which is freely available for redistribution 